\title{
The set of solutions of a polynomial-exponential equation
}

\author{
by \\ Scott Ahlgren (University Park, PA)
}

1. Introduction. In this paper we shall study equations of polynomialexponential type defined over the algebraic numbers. These are equations of the form

$$
\sum_{l=1}^{k} P_{l}(\boldsymbol{x}) \boldsymbol{\alpha}_{l}^{x}=0
$$

in variables $\boldsymbol{x}=\left(x_{1}, \ldots, x_{n}\right) \in \mathbb{Z}^{n}$, where the $P_{l}$ are polynomials with coefficients in a number field $K$ and

$$
\boldsymbol{\alpha}_{l}^{x}=\alpha_{l 1}^{x_{1}} \ldots \alpha_{l n}^{x_{n}}
$$

with $\alpha_{l j} \in K^{\times}(1 \leq l \leq k, 1 \leq j \leq n)$. We begin with some basic notation. Set $\Lambda=\{1, \ldots, k\}$. When $\mathcal{P}$ is a partition of $\Lambda$, we will write $\lambda \in \mathcal{P}$ to mean that $\lambda$ is one of the subsets of $\Lambda$ appearing in $\mathcal{P}$. We then consider the system of equations

$$
\sum_{l \in \lambda} P_{l}(\boldsymbol{x}) \boldsymbol{\alpha}_{l}^{x}=0 \quad(\lambda \in \mathcal{P}) .
$$

Denote by $S(\mathcal{P})$ the set of solutions of $(1.1)_{\mathcal{P}}$ which do not satisfy $(1.1)_{\mathcal{Q}}$ for any proper refinement $\mathcal{Q}$ of $\mathcal{P}$ (notice that every solution of (1.1) lies in $S(\mathcal{P})$ for some partition $\mathcal{P})$. Then let $G(\mathcal{P})$ be the subgroup of $\mathbb{Z}^{n}$ consisting of $\boldsymbol{x}$ such that

$$
\boldsymbol{\alpha}_{l}^{x}=\boldsymbol{\alpha}_{m}^{x}
$$

whenever $l$ and $m$ lie in the same set $\lambda$ of $\mathcal{P}$.

Let $d$ be the degree of the field $K$, and for $l \in \Lambda$ let $\delta_{l}$ be the total degree of the polynomial $P_{l}$. Set

$$
A=\sum_{l \in \Lambda}\left(\begin{array}{c}
n+\delta_{l} \\
n
\end{array}\right), \quad B=\max (n, A) .
$$

1991 Mathematics Subject Classification: Primary 11D61. 
$A$ is the potential number of non-zero coefficients of the polynomials $P_{1}, \ldots$ $\ldots, P_{k}$, and $B=A$ unless all of these polynomials are constant, in which case $B=\max (n, k)$. Recently, Schlickewei and Schmidt [7] have proved the following

Theorem $1.1[7$, Theorem 1]. If $G(\mathcal{P})=\{\mathbf{0}\}$ then

$$
|S(\mathcal{P})|<2^{35 B^{3}} d^{6 B^{2}} \text {. }
$$

This improves (both with respect to the size and to the dependence on certain parameters) the bound which the same authors obtained in [6].

The object in this paper is to study the case when the group $G(\mathcal{P})$ is non-trivial. Let $|\cdot|$ be the euclidean norm on $\mathbb{R}^{n}$ and, for positive $z$, define $\log ^{+} z=\max (\log z, 1)$. Let $r$ be the rank of $G(\mathcal{P})$ and let $H$ be the $r$-dimensional subspace of $\mathbb{R}^{n}$ spanned by $G(\mathcal{P})$. Let $H^{\perp}$ denote the orthogonal complement of $H$. Then any $\boldsymbol{\xi} \in \mathbb{R}^{n}$ may be written uniquely as a sum

$$
\boldsymbol{\xi}=\boldsymbol{\xi}^{H}+\boldsymbol{\xi}^{\perp}
$$

where $\boldsymbol{\xi}^{H} \in H$ and $\boldsymbol{\xi}^{\perp} \in H^{\perp}$. Set

$$
c_{0}=B^{12} 2^{4 B+21} d^{6},
$$

and define

$$
\mathcal{S}=\left\{\boldsymbol{x} \in \mathbb{Z}^{n}:\left|\boldsymbol{x}^{\perp}\right| \leq c_{0} \log ^{+}\left|\boldsymbol{x}^{H}\right|\right\} .
$$

Let $\mathbb{G}$ be the group of transformations of $\mathbb{R}^{n}$ of the form

$$
\phi \boldsymbol{x}=\Theta \boldsymbol{x}+\boldsymbol{u},
$$

where $\Theta \in \operatorname{GL}(n, \mathbb{Z})$ fixes $H$ pointwise, and $\boldsymbol{u} \in \mathbb{Z}^{n}$. Our main result is

THEOREM 1.2. With notation as above, we have

$$
S(\mathcal{P}) \subseteq \bigcup_{\phi \in \Phi} \phi(\mathcal{S})
$$

where $\Phi \subset \mathbb{G}$ and $|\Phi| \leq 2^{36 B^{3}} d^{6 B^{2}}$.

Previously, Laurent [3] (see also [4]) had obtained qualitative versions of Theorems 1.1 and 1.2. It should be noted that the map $\phi \mapsto \phi(\mathcal{S})$ is not one-to-one. To be precise, write $\phi_{1} \sim \phi_{2}$ if $\phi_{i} \boldsymbol{x}=\Theta_{i} \boldsymbol{x}+\boldsymbol{u}_{i}(i=1,2)$, where $\boldsymbol{u}_{1}=\boldsymbol{u}_{2}$ and $\Theta_{1}, \Theta_{2}$ induce the same map on $\mathbb{R}^{n} / H$. Then $\phi_{1}(\mathcal{S})=\phi_{2}(\mathcal{S})$ whenever $\phi_{1} \sim \phi_{2}$.

2. Absolute values and heights. We briefly describe some facts about heights which will be used throughout. When $K$ is a number field, let $V=$ $V(K)$ denote the set of places of $K$. For $v \in V(K)$ let $|\cdot|_{v}$ be the associated absolute value, normalized to extend the usual, or a $p$-adic absolute value of $\mathbb{Q}$, and let $\|\cdot\|_{v}=|\cdot|_{v}^{d_{v} / d}$, where $d$ is the degree of $K$ and $d_{v}$ is the local 
degree. For $\boldsymbol{\alpha}=\left(\alpha_{1}, \ldots, \alpha_{n}\right) \in K^{n}$ define the absolute multiplicative height $H(\boldsymbol{\alpha})$ by

$$
H(\boldsymbol{\alpha})=\prod_{v \in V}\|\boldsymbol{\alpha}\|_{v}
$$

where $\|\boldsymbol{\alpha}\|_{v}=\max \left(\left\|\alpha_{1}\right\|_{v}, \ldots,\left\|\alpha_{n}\right\|_{v}\right)$. Then define the absolute logarithmic height $h(\boldsymbol{\alpha})=\log H(\boldsymbol{\alpha})$. Both heights are defined on $\mathbb{P}^{n-1}(\mathbb{A})$, where $\mathbb{A}$ is the field of algebraic numbers.

When $\alpha \in K$ set $H(\alpha)=H(1, \alpha)$ and $h(\alpha)=h(1, \alpha)$. Then

$$
h(\alpha)=\sum_{v \in V} \max \left(0, \log \|\alpha\|_{v}\right)=\frac{1}{2} \sum_{v \in V}\left|\log \|\alpha\|_{v}\right| .
$$

As pointed out in [6], it is a consequence of Dobrowolski's work [2] that if $\alpha$ has degree $d$ and is neither zero nor a root of unity, then

$$
h(\alpha)>\left(21 d^{3}\right)^{-1} \text {. }
$$

3. The first step. The proof of Theorem 1.2 generalizes the method developed by Schlickewei and Schmidt in [7]. In the next two sections, we shall accomplish two separate goals. In the present section we give a theorem of Schlickewei and Schmidt which lies at the heart of the method, and in the following, we use an induction argument to reduce the proof of Theorem 1.2 to that of a certain Theorem 4.1. The task for the remainder of the paper, then, will be to prove Theorem 4.1; in Section 5 we shall pause again to give an overview of its proof.

A fundamental step will be to consider (1.1) as a linear equation in a large-dimensional space over the field $K$. To develop this idea requires some notation. In (1.1), let $\mathcal{M}_{l}$ be the set of monomials of total degree $\leq \delta_{l}$. We may then write

$$
P_{l}=\sum_{M \in \mathcal{M}_{l}} a_{l M} M
$$

with coefficients $a_{l M} \in K$. Define

$$
\mathcal{A}=\left\{(l, M): l \in \Lambda, M \in \mathcal{M}_{l}, a_{l M} \neq 0\right\} .
$$

Then (1.1) may be written as

$$
\sum_{(l, M) \in \mathcal{A}} a_{l M} M(\boldsymbol{x}) \boldsymbol{\alpha}_{l}^{\boldsymbol{x}}=0 .
$$

Now define

$$
\eta_{l M}(\boldsymbol{x})=a_{l M} M(\boldsymbol{x}) \boldsymbol{\alpha}^{x},
$$

and let $\boldsymbol{\eta}(\boldsymbol{x})$ be the vector with components $\eta_{l M}(\boldsymbol{x})((l, M) \in \mathcal{A})$. Define 
$a=|\mathcal{A}|$; then the vector $\boldsymbol{\eta}(\boldsymbol{x})$ lies in $K^{a}$. The equation (3.1) becomes

$$
\sum_{(l, M) \in \mathcal{A}} \eta_{l M}(\boldsymbol{x})=0
$$

This asserts that $\boldsymbol{\eta}(\boldsymbol{x})$ lies in a certain subspace $T$ of $K^{a}$ of codimension 1.

Let $h_{M}(\boldsymbol{x})$ be the logarithmic height of the vector with components $M(\boldsymbol{x})\left(M \in \mathcal{M}_{1} \cup \ldots \cup \mathcal{M}_{k}\right)$, and let $h_{E}(\boldsymbol{x})$ be the height of the vector with components $a_{l M} \boldsymbol{\alpha}_{l}^{x}((l, M) \in \mathcal{A})$.

We shall require the following result of Schlickewei and Schmidt.

Theorem 3.1 [7, Proposition B]. Suppose that $a \geq 3$. Then as $\boldsymbol{x}$ ranges over solutions of (3.2) with

$$
h_{M}(\boldsymbol{x}) \leq \frac{1}{4 a^{2}} h_{E}(\boldsymbol{x})
$$

the vector $\boldsymbol{\eta}(\boldsymbol{x})$ will be contained in the union of not more than

$$
2^{30 a^{2}}\left(32 a^{2}\right)^{n} d^{3(n+a)}
$$

proper subspaces of $T$.

This theorem will be the basic tool in the proof of Theorem 1.2. A priori, since $h_{M}$ involves monomials and $h_{E}$ involves exponentials, it would seem that (3.3) should be easy to achieve. However, at the outset we have no control over the size of the coefficients $a_{l M}$ in $h_{M}$, and it is essential that our bounds are independent of these coefficients. Hence much work will be required to produce the inequality (3.3); this is the subject of Sections 8 through 11 .

4. A reduction. To discuss the aforementioned Theorem 4.1, we must introduce further notation. Recall the definition (1.2) of $A$. We will consider vectors $\boldsymbol{\xi} \in K^{A}$ with components $\xi_{l M}$ indexed by pairs $l, M$ with $l \in \Lambda$, $M \in \mathcal{M}_{l}$.

When $\lambda \subseteq \Lambda$, let $V_{\lambda}$ be the coordinate subspace of $K^{A}$ consisting of vectors $\boldsymbol{\xi}$ with $\xi_{l M}=0$ when $l \notin \lambda$. Then for any partition $\mathcal{Q}$, we have

$$
\sum_{\lambda \in \mathcal{Q}} V_{\lambda}=K^{A} \text {. }
$$

When $T$ is a subspace of $K^{A}$ and $\mathcal{Q}$ is a partition of $\Lambda$, let $T(\mathcal{Q})$ be the subspace of $T$ defined by

$$
T(\mathcal{Q})=\sum_{\lambda \in \mathcal{Q}} T \cap V_{\lambda}
$$

If $\mathcal{Q}^{\prime}$ is a refinement of $\mathcal{Q}$ then $T\left(\mathcal{Q}^{\prime}\right) \subseteq T(\mathcal{Q})$. Therefore $T\left(\mathcal{Q}^{\prime}\right)=T \Rightarrow$ $T(\mathcal{Q})=T$. Write $\mathcal{Q} \prec T$ if $T(\mathcal{Q})=T$ but no proper refinement $\mathcal{Q}^{\prime}$ of $\mathcal{Q}$ has $T\left(\mathcal{Q}^{\prime}\right)=T$. For any $T$ there exists $\mathcal{Q}$ (not necessarily unique) with $\mathcal{Q} \prec T$. 
For each $l \in \Lambda$, let $P_{l}$ be a polynomial of total degree $\leq \delta_{l}$. We may write $P_{l}=\sum_{M \in \mathcal{M}_{l}} a_{l M} M$. Given $\boldsymbol{x} \in \mathbb{Z}^{n}$, let $\boldsymbol{\xi}=\boldsymbol{\xi}(\boldsymbol{x}) \in K^{A}$ be the vector with components

$$
\xi_{l M}=\xi_{l M}(\boldsymbol{x})=M(\boldsymbol{x}) \boldsymbol{\alpha}_{l}^{x} \quad\left(l \in \Lambda, M \in \mathcal{M}_{l}\right) .
$$

Then to say that $\boldsymbol{x}$ satisfies $(1.1)_{\mathcal{P}}$ is the same as to say that $\boldsymbol{\xi}(\boldsymbol{x})$ satisfies the system

$$
\sum_{l \in \lambda} \sum_{M \in \mathcal{M}_{l}} a_{l M} \xi_{l M}=0 \quad(\lambda \in \mathcal{P}) .
$$

The equations (4.1) define a certain subspace, say $W$, of $K^{A}$.

When $T$ is any subspace of $K^{A}$, let $\mathfrak{X}(T)$ consist of $\boldsymbol{x} \in \mathbb{Z}^{n}$ with $\boldsymbol{\xi}(\boldsymbol{x}) \in T$. If $\mathcal{Q}$ is a partition of $\Lambda$, let $\mathfrak{X}(T, \mathcal{Q})$ consist of $\boldsymbol{x} \in \mathbb{Z}^{n}$ with $\boldsymbol{\xi}(\boldsymbol{x}) \in T(\mathcal{Q})$, but $\boldsymbol{\xi}(\boldsymbol{x}) \notin T\left(\mathcal{Q}^{\prime}\right)$ for any proper refinement $\mathcal{Q}^{\prime}$ of $\mathcal{Q}$. With $S(\mathcal{P})$ as defined in Section 1, we have

$$
S(\mathcal{P})=\mathfrak{X}(W, \mathcal{P}),
$$

where $W$ is defined by (4.1). Notice that if $\mathcal{P}=\{\{1\}, \ldots,\{k\}\}$ then $G(\mathcal{P})=$ $\mathbb{Z}^{n}$ and Theorem 1.2 holds trivially. Therefore we shall always suppose that $\mathcal{P}$ is not this partition into singletons. The next result is an analogue of Proposition C of [7].

THEOREM 4.1. Set $C=2^{35 B^{2}} d^{6 B}$. Let $T$ be a non-zero subspace of $K^{A}$ with $\mathcal{P} \prec T$. Then there exist $\phi \in \mathbb{G}$ and proper subspaces $T_{i}$ of $T$ with $T_{i}(\mathcal{P})=T_{i}(i=1, \ldots, C)$ such that

$$
\mathfrak{X}(T, \mathcal{P}) \subseteq \phi(\mathcal{S}) \cup \bigcup_{i=1}^{C} \mathfrak{X}\left(T_{i}\right) .
$$

In the remainder of this section we show that Theorem 4.1 implies Theorem 1.2 ; we begin with a lemma.

Lemma 4.2. Suppose that Theorem 4.1 is true, and suppose that $T$ is a subspace of $K^{A}$ with $\mathcal{P} \prec T$ and $\operatorname{dim} T=t$. Then

$$
\mathfrak{X}(T, \mathcal{P}) \subseteq \bigcup_{\phi \in \Phi_{T}} \phi(\mathcal{S}),
$$

where $\Phi_{T} \subset \mathbb{G}$ and

$$
\left|\Phi_{T}\right| \leq(2 C)^{t}
$$

Proof. This is proved by induction on $t$. When $t=0, \mathfrak{X}(T, \mathcal{P})$ is empty (notice that $\boldsymbol{\xi}(\boldsymbol{x})=\mathbf{0}$ is impossible since when $M=1$, we have $\xi_{l M}(\boldsymbol{x})=$ $\left.\boldsymbol{\alpha}_{l}^{x}\right)$. Suppose, then, that $t>0$. Let

$$
\mathfrak{X}(T, \mathcal{P}) \subseteq \phi(\mathcal{S}) \cup \bigcup_{i=1}^{C} \mathfrak{X}\left(T_{i}\right)
$$


be the cover given by Theorem 4.1. Suppose that $\mathcal{P} \prec T_{i}$ fails to hold for some index $i$. Then there is a proper refinement $\mathcal{Q}$ of $\mathcal{P}$ with $T_{i}(\mathcal{Q})=T_{i}$, so that $\mathfrak{X}\left(T_{i}\right)=\mathfrak{X}\left(T_{i}(\mathcal{Q})\right) \subseteq \mathfrak{X}(T(\mathcal{Q}))$ has empty intersection with $\mathfrak{X}(T, \mathcal{P})$. Suppose on the other hand that $\mathcal{P} \prec T_{i}$. Then if $\boldsymbol{x} \in \mathfrak{X}(T, \mathcal{P}) \cap \mathfrak{X}\left(T_{i}\right)$, we have $\boldsymbol{\xi}(\boldsymbol{x}) \in T_{i}=T_{i}(\mathcal{P})$. Since $\boldsymbol{x} \in \mathfrak{X}(T, P)$, we cannot have $\boldsymbol{\xi}(\boldsymbol{x}) \in T_{i}(\mathcal{Q}) \subseteq T(\mathcal{Q})$ for a proper refinement $\mathcal{Q}$ of $\mathcal{P}$, so that $\boldsymbol{x} \in \mathfrak{X}\left(T_{i}, \mathcal{P}\right)$. In this case, we obtain

$$
\mathfrak{X}(T, \mathcal{P}) \cap \mathfrak{X}\left(T_{i}\right) \subseteq \mathfrak{X}\left(T_{i}, \mathcal{P}\right) .
$$

In light of this discussion, we may rewrite (4.2) as

$$
\mathfrak{X}(T, \mathcal{P}) \subseteq \phi(\mathcal{S}) \cup \bigcup_{\mathcal{P} \prec T_{i}} \mathfrak{X}\left(T_{i}, \mathcal{P}\right) .
$$

By the induction hypothesis, each set $\mathfrak{X}\left(T_{i}, \mathcal{P}\right)$ is contained in the union of not more than $(2 C)^{t-1}$ sets $\phi(\mathcal{S})$. Therefore $(4.3)$ shows that $\mathfrak{X}(T, \mathcal{P})$ is contained in the union of not more than $(2 C)^{t}$ such sets.

Theorem 1.2 follows easily from this lemma. Recall that $S(\mathcal{P})=\mathfrak{X}(W, \mathcal{P})$, where $W$ is the subspace defined in (4.1). Notice that $\operatorname{dim} W \leq A \leq B$. If $\mathcal{P} \prec W$ fails to hold, then, as above, $S(\mathcal{P})=\mathfrak{X}(W, \mathcal{P})$ is empty. If, however, $\mathcal{P} \prec W$, then the lemma shows that $S(\mathcal{P})$ is contained in the union of not more than $(2 C)^{B} \leq 2^{36 B^{3}} d^{6 B^{2}}$ sets $\phi(\mathcal{S})$, and the theorem is proved.

Our only remaining task is to prove Theorem 4.1.

5. The plan of attack. It will be useful at this point to give a vague outline of the method which we shall employ to prove Theorem 4.1. We begin in the next section by showing that in order to prove our theorem, we may first apply a transformation $\phi \in \mathbb{G}$ to the variable vector $\boldsymbol{x}$. Then, given a subspace $T$ as in the theorem, we shall require an assortment $\{L\}$ of suitable linear forms which vanish on $T$ (a suitable form, among other things, must behave appropriately under the action of the aforementioned transformations). The method of constructing such forms was developed in [7], and will be outlined in Section 9 .

When $\boldsymbol{x} \in \mathfrak{X}(T, \mathcal{P})$ and $L$ vanishes on $T$ we have $L(\boldsymbol{\xi}(\boldsymbol{x}))=0$. This is an equation of the form (3.2), and our plan will be to apply Theorem 3.1, which assumes a certain inequality (3.3) involving heights. In an attempt to produce this inequality we will apply two transformations $\phi \in \mathbb{G}$ to $\boldsymbol{x}$; this is the subject of Sections 8 and 10. As it turns out, every $\boldsymbol{x}$ for which we cannot produce (3.3) will lie in $\mathcal{S}$. When $\boldsymbol{x}$ does have (3.3), Theorem 3.1 will show that $\boldsymbol{\xi}(\boldsymbol{x})$ lies in one of finitely many proper subspaces $T_{i}$ of $T$, from which we will obtain an assertion of the form given in Theorem 4.1. To achieve this reduction of dimension it will be essential that our linear forms $L$ are minimal; that is, roughly speaking, that they have the smallest possible set of non-zero coefficients. 
6. Transformations. Recall the definition of the group $\mathbb{G}$ given in the introduction. In this section we show that in order to prove Theorem 4.1, we may first apply a transformation $\phi \in \mathbb{G}$ to the variable vector $\boldsymbol{x}$. Write $\phi \boldsymbol{x}=\Theta \boldsymbol{x}+\boldsymbol{u}$ as in the introduction. Define

$$
\beta_{l j}=\boldsymbol{\alpha}_{l}^{\Theta e_{j}} \quad(l \in \Lambda, 1 \leq j \leq n),
$$

where $\boldsymbol{e}_{1}, \ldots, \boldsymbol{e}_{n}$ are the standard basis vectors, and let $\boldsymbol{\beta}_{l}=\left(\beta_{l 1}, \ldots, \beta_{l n}\right)$ $(l \in \Lambda)$. Then for each $l$ we have $\boldsymbol{\alpha}_{l}^{\Theta x}=\boldsymbol{\beta}_{l}^{x}$. Recall that $\boldsymbol{\xi}(\boldsymbol{x})$ was defined in terms of the $\boldsymbol{\alpha}_{l}$; to indicate this write $\boldsymbol{\xi}(\boldsymbol{x})=\boldsymbol{\xi}^{\alpha}(\boldsymbol{x})$. Then let $\boldsymbol{\xi}^{\beta}(\boldsymbol{x})$ be the vector with components

$$
M(\boldsymbol{x}) \boldsymbol{\beta}_{l}^{x} \quad\left(l \in \Lambda, M \in \mathcal{M}_{l}\right) .
$$

In $[6, \S 7]$ it is shown that $\phi$ induces an invertible linear map $\widehat{\phi}$ on $K^{A}$ with the property that

$$
\boldsymbol{\xi}^{\alpha}(\phi \boldsymbol{x})=\widehat{\phi} \boldsymbol{\xi}^{\beta}(\boldsymbol{x}) .
$$

Write $G(\mathcal{P})=G^{\alpha}(\mathcal{P}), \mathfrak{X}(T)=\mathfrak{X}^{\alpha}(T), \mathfrak{X}(T, \mathcal{P})=\mathfrak{X}^{\alpha}(T, \mathcal{P})$, and define $G^{\beta}(\mathcal{P}), \mathfrak{X}^{\beta}(T)$, and $\mathfrak{X}^{\beta}(T, \mathcal{P})$ as before, but with respect to the $\boldsymbol{\beta}_{l}$. For any partition $\mathcal{P}$ and subspace $T$ of $K^{A}$ the following properties were derived in $[6]$ :

$$
\begin{aligned}
\mathfrak{X}^{\alpha}(\widehat{\phi} T) & =\phi \mathfrak{X}^{\beta}(T), \\
\mathfrak{X}^{\alpha}(\widehat{\phi} T, \mathcal{P}) & =\phi \mathfrak{X}^{\beta}(T, \mathcal{P}), \\
\mathcal{P} \prec T & \Leftrightarrow \mathcal{P} \prec \widehat{\phi} T, \\
\widehat{\phi}(T(\mathcal{P})) & =(\widehat{\phi} T)(\mathcal{P}), \\
G^{\alpha}(\mathcal{P}) & =\Theta G^{\beta}(\mathcal{P}) .
\end{aligned}
$$

From (6.6) and the definition of the group $\mathbb{G}$, we see that in fact,

$$
G^{\beta}(\mathcal{P})=G^{\alpha}(\mathcal{P}),
$$

so that the subspace $H$ is preserved by such a transformation.

Suppose now that we have established the assertion in Theorem 4.1 after applying the transformation $\phi \in \mathbb{G}$ to $\boldsymbol{x}$ (this involves replacing the $\boldsymbol{\alpha}_{l}$ by $\boldsymbol{\beta}_{l}$ as above). In other words, suppose that we have proved the following assertion:

Whenever $T$ is a subspace with $T \prec \mathcal{P}$, there exist $\psi \in \mathbb{G}$ and proper subspaces $T_{i}$ of $T(i=1, \ldots, C)$ with $T_{i}(\mathcal{P})=T_{i}$ and such that

$$
\mathfrak{X}^{\beta}(T, \mathcal{P}) \subseteq \psi(\mathcal{S}) \cup \bigcup_{i=1}^{C} \mathfrak{X}^{\beta}\left(T_{i}\right) .
$$


Our goal is to show that this implies the same assertion with $\beta$ replaced by $\alpha$. Define $W=\widehat{\phi} T$ and $W_{i}=\widehat{\phi} T_{i}$. If we use (6.2) and (6.3), (6.8) becomes

$$
\phi^{-1} \mathfrak{X}^{\alpha}(W, \mathcal{P}) \subseteq \psi(\mathcal{S}) \cup \bigcup_{i=1}^{C} \phi^{-1} \mathfrak{X}^{\alpha}\left(W_{i}\right),
$$

which is the same as

$$
\mathfrak{X}^{\alpha}(W, \mathcal{P}) \subseteq \phi \psi(\mathcal{S}) \cup \bigcup_{i=1}^{C} \mathfrak{X}^{\alpha}\left(W_{i}\right) .
$$

By (6.4), we have $W \prec \mathcal{P} \Leftrightarrow T \prec \mathcal{P}$, and by (6.5) we have

$$
W_{i}(\mathcal{P})=\left(\widehat{\phi} T_{i}\right)(\mathcal{P})=\widehat{\phi}\left(T_{i}(\mathcal{P})\right)=\widehat{\phi}\left(T_{i}\right)=W_{i} .
$$

Therefore our goal is achieved; after this discussion, we reach the following conclusion:

In order to prove Theorem 4.1, we may first apply a transformation $\phi \in \mathbb{G}$ to the variable vector $\boldsymbol{x}$.

7. Linear forms. We collect further notation from [7] which we shall require. Any linear form $L$ on $K^{A}$ may be written

$$
L(\boldsymbol{\xi})=\sum_{l \in \Lambda} \sum_{M \in \mathcal{M}_{l}} b_{l M} \xi_{l M}=L^{1}\left(\boldsymbol{\xi}_{1}\right)+\ldots+L^{k}\left(\boldsymbol{\xi}_{k}\right),
$$

where $\boldsymbol{\xi}=\left(\boldsymbol{\xi}_{1}, \ldots, \boldsymbol{\xi}_{k}\right), \boldsymbol{\xi}_{l}=\left(\xi_{l M}\right)_{M \in \mathcal{M}_{l}}$, and

$$
L^{l}\left(\boldsymbol{\xi}_{l}\right)=\sum_{M \in \mathcal{M}_{l}} b_{l M} \xi_{l M}
$$

with $b_{l M} \in K$. Set

$$
\mathcal{B}(L)=\left\{l \in \Lambda: L^{l} \neq 0\right\}, \quad \mathcal{A}(L)=\left\{(l, M): b_{l M} \neq 0\right\} .
$$

Let $T$ be the subspace of $K^{A}$ given in Theorem 4.1, and let $\mathcal{L}(T)$ be the space of linear forms vanishing on $T$. Then $\mathcal{L}(T) \neq\{0\}$ (for if $\mathcal{L}(T)=\{0\}$, then $T=K^{A}$, so that $\mathcal{P} \prec T$ implies that $\mathcal{P}$ is the partition consisting entirely of singletons). A non-zero form $L \in \mathcal{L}(T)$ will be called minimal if there is no non-zero form $L^{\prime} \in \mathcal{L}(T)$ with $\mathcal{A}\left(L^{\prime}\right) \varsubsetneqq \mathcal{A}(L)$. Since $\mathcal{P} \prec T$, a minimal form $L$ has $\mathcal{B}(L) \subseteq \lambda$ for some $\lambda \in \mathcal{P}$.

We introduce a lexicographical ordering of monomials: write $M>N$ if $M=X_{1}^{i_{1}} \ldots X_{n}^{i_{n}}, N=X_{1}^{j_{1}} \ldots X_{n}^{j_{n}}$ with $i_{s}>j_{s}, i_{s+1}=j_{s+1}, \ldots, i_{n}=j_{n}$ for some $s$. We also introduce a symbol $\square$ such that $M>\square$ for every monomial $M$. Let $L$ be a form, written as in (7.1). If $l \in \mathcal{B}(L)$, then $L^{l} \neq 0$. In this case we let $M_{l}(L)$ be the largest (with respect to $>$ ) monomial with non-zero coefficient $b_{l M}$, and let $b_{l}(L)$ be the corresponding coefficient. If $l \notin \mathcal{B}(L)$, then $L^{l}=0$, and we set $M_{l}(L)=\square, b_{l}(L)=0$, and $b_{l}(L) M_{l}(L)=\square$. 
With every form $L$ we associate $k$-tuples of leading monomials, leading coefficients, and leading terms: $\left(M_{1}(L), \ldots, M_{k}(L)\right),\left(b_{1}(L), \ldots, b_{k}(L)\right)$, and $\left(b_{1}(L) M_{1}(L), \ldots, b_{k}(L) M_{k}(L)\right)$, respectively.

8. The first transformation. In this section we shall make the first step towards producing the inequality (3.3). Recall that this inequality required that the height of a vector with components $a_{l M} \boldsymbol{\alpha}_{l}^{x}$ be large. We begin here by considering certain vectors with components $\boldsymbol{\alpha}_{l}^{x}$, and we show that after an appropriate transformation the height of such vectors can be controlled.

Let $\boldsymbol{\alpha}, \boldsymbol{\beta} \in\left(K^{\times}\right)^{n}$ have components $\alpha_{j}, \beta_{j}$, respectively. We define $\boldsymbol{\alpha} / \boldsymbol{\beta}=\left(\alpha_{1} / \beta_{1}, \ldots, \alpha_{n} / \beta_{n}\right)$. Now, given $\boldsymbol{\alpha}_{1}, \ldots, \boldsymbol{\alpha}_{k}$ as in Section 1 , define $\boldsymbol{\alpha}^{l m}=\boldsymbol{\alpha}_{l} / \boldsymbol{\alpha}_{m}(1 \leq l, m \leq k)$, and let $\alpha_{j}^{l m}$ be the $j$ th component of $\boldsymbol{\alpha}^{l m}$. Set

$$
\gamma_{j v}^{l m}=\log \left\|\alpha_{j}^{l m}\right\|_{v} \quad(1 \leq j \leq n, v \in V(K)) .
$$

Then $\sum_{v} \gamma_{j v}^{l m}=0$ by the product formula (here, and below, a sum over $v$ will mean a sum over $v \in V(K))$. For $\boldsymbol{\xi}=\left(\xi_{1}, \ldots, \xi_{n}\right) \in \mathbb{R}^{n}$, define

$$
g_{v}^{l m}(\boldsymbol{\xi})=\sum_{j=1}^{n} \gamma_{j v}^{l m} \xi_{j}
$$

Notice that $\sum_{v} g_{v}^{l m}(\boldsymbol{\xi})=0$. Then define

$$
\psi_{l m}(\boldsymbol{\xi})=\sum_{v} \max \left(0, g_{v}^{l m}(\boldsymbol{\xi})\right)=\frac{1}{2} \sum_{v}\left|g_{v}^{l m}(\boldsymbol{\xi})\right| .
$$

For $\boldsymbol{x} \in \mathbb{Z}^{n}$, we have $g_{v}^{l m}(\boldsymbol{x})=\log \left\|\left(\boldsymbol{\alpha}_{l} / \boldsymbol{\alpha}_{m}\right)^{x}\right\|_{v}$, whence

$$
\psi_{l m}(\boldsymbol{x})=h\left(\left(\boldsymbol{\alpha}_{l} / \boldsymbol{\alpha}_{m}\right)^{\boldsymbol{x}}\right)=h\left(\boldsymbol{\alpha}_{l}^{\boldsymbol{x}}, \boldsymbol{\alpha}_{m}^{x}\right) \quad\left(\boldsymbol{x} \in \mathbb{Z}^{n}\right) .
$$

Notice that for $1 \leq l, m, p \leq k$, we have $\gamma_{j v}^{l m}=\gamma_{j v}^{l p}+\gamma_{j v}^{p m}$. It follows that

$$
\psi_{l m}(\boldsymbol{\xi}) \leq \psi_{l p}(\boldsymbol{\xi})+\psi_{p m}(\boldsymbol{\xi}) \quad\left(\boldsymbol{\xi} \in \mathbb{R}^{n}\right) ;
$$

this fact will be needed in the next section.

Recall that we write $l \stackrel{\mathcal{P}}{\sim} m$ to mean that $l$ and $m$ lie in the same set $\lambda$ of $\mathcal{P}$. For $v \in V(K)$ define

$$
N_{v}=\left\{\boldsymbol{\xi}: g_{v}^{l m}(\boldsymbol{\xi})=0 \text { whenever } l \stackrel{\mathcal{D}}{\sim} m\right\}
$$

so that $N_{v}$ is a subspace of $\mathbb{R}^{n}$. When $\boldsymbol{x} \in \mathbb{Z}^{n}$, we have

$$
g_{v}^{l m}(\boldsymbol{x})=\log \left\|\left(\boldsymbol{\alpha}_{l} / \boldsymbol{\alpha}_{m}\right)^{x}\right\|_{v} .
$$

Therefore $G(\mathcal{P})$ is contained in $N_{v}$, so that $H \subseteq N_{v}$ for each $v \in V(K)$. This shows that when $l \stackrel{\mathcal{P}}{\sim} m$, we have $\psi_{l m}(\boldsymbol{\xi}+\boldsymbol{h})=\psi_{l m}(\boldsymbol{\xi})$ for any $\boldsymbol{\xi} \in \mathbb{R}^{n}$ and $\boldsymbol{h} \in H$.

As in the introduction, let $H^{\perp}$ be the orthogonal complement of $H ; H^{\perp}$ is a subspace of dimension $s=n-r$. If $n=r$ then $\mathcal{S}=\mathbb{Z}^{n}$ and Theorem 4.1 
holds trivially; we may therefore suppose that $s>0$. Recall that when $\boldsymbol{\xi} \in \mathbb{R}^{n}$ we write $\boldsymbol{\xi}=\boldsymbol{\xi}^{H}+\boldsymbol{\xi}^{\perp}$ where $\boldsymbol{\xi}^{H} \in H$ and $\boldsymbol{\xi}^{\perp} \in H^{\perp}$.

Lemma 8.1. Let $\Gamma$ be the projection of $\mathbb{Z}^{n}$ on $H^{\perp}$. Then $\Gamma$ is a lattice on $H^{\perp}$.

Proof. Let $\pi: \mathbb{Z}^{n} \rightarrow H^{\perp}$ denote the projection map. It is clear that $\pi\left(\mathbb{Z}^{n}\right)$ is a group under addition. Since $H^{\perp}$ is a rational subspace we know that $H^{\perp} \cap \mathbb{Z}^{n} \subseteq \pi\left(\mathbb{Z}^{n}\right)$ is a lattice on $H^{\perp}$; therefore $\pi\left(\mathbb{Z}^{n}\right)$ contains $s$ linearly independent points. So to prove our lemma we need only show that $\pi\left(\mathbb{Z}^{n}\right)$ is discrete. Since $H^{\perp}$ is a rational subspace, there exists a basis of $\mathbb{R}^{n}$ of the form $\left\{\boldsymbol{a}_{i}\right\}_{1 \leq i \leq n}$, where each $\boldsymbol{a}_{i}$ has integral components, and further, we have $\boldsymbol{a}_{i} \in H^{\perp}(1 \leq i \leq s)$ and $\boldsymbol{a}_{i} \in H(s+1 \leq i \leq n)$. Let $\boldsymbol{e}_{1}, \ldots, \boldsymbol{e}_{n}$ be the standard basis vectors. Then there exist rationals $\alpha_{i j}$ such that $\boldsymbol{e}_{j}=$ $\sum_{i=1}^{n} \alpha_{i j} \boldsymbol{a}_{j}(1 \leq j \leq n)$. If $D$ is a common denominator for all of the $\alpha_{i j}$, then we see that for each $j$ we have

$$
\pi\left(D \boldsymbol{e}_{j}\right)=\sum_{i=1}^{s}\left(D \alpha_{i j}\right) \boldsymbol{a}_{j} \in H^{\perp} \cap \mathbb{Z}^{n} .
$$

It follows that $\pi\left(\mathbb{Z}^{n}\right) \subseteq \frac{1}{D}\left(H^{\perp} \cap \mathbb{Z}^{n}\right)$. Since $H^{\perp} \cap \mathbb{Z}^{n}$ is discrete we conclude that $\pi\left(\mathbb{Z}^{n}\right)$ is discrete, and the lemma is proved.

Now let $\mathcal{P}$ be a partition as in Theorem 4.1. Define the function

$$
\omega^{\mathcal{P}}(\boldsymbol{\xi})=\max _{\lambda \in \mathcal{P}} \max _{l, m \in \lambda} \psi_{l m}(\boldsymbol{\xi}) .
$$

Since $\mathcal{P}$ is fixed, we will write $\omega=\omega^{\mathcal{P}}$ for simplicity. For any $\boldsymbol{\xi}$, the discussion above shows that $\psi_{l m}\left(\boldsymbol{\xi}^{\perp}\right)=\psi_{l m}(\boldsymbol{\xi})$; therefore also $\omega\left(\boldsymbol{\xi}^{\perp}\right)=\omega(\boldsymbol{\xi})$.

When $\boldsymbol{x} \in \mathbb{Z}^{n}, \boldsymbol{x} \notin H$, there exist $l, m$ with $l \stackrel{\mathcal{P}}{\sim} m$ such that $\left(\boldsymbol{\alpha}_{l} / \boldsymbol{\alpha}_{m}\right)^{\boldsymbol{x}}$ is not a root of unity. Using (8.6), (8.4), and Dobrowolski's estimate (2.1), we see that

$$
\omega\left(\boldsymbol{x}^{\perp}\right) \geq\left(21 d^{3}\right)^{-1} \quad\left(\boldsymbol{x}^{\perp} \in \Gamma, \boldsymbol{x}^{\perp} \neq \mathbf{0}\right) .
$$

From (8.3) we infer the properties

$$
\begin{gathered}
\omega\left(\boldsymbol{\xi}^{\perp}+\boldsymbol{\eta}^{\perp}\right) \leq \omega\left(\boldsymbol{\xi}^{\perp}\right)+\omega\left(\boldsymbol{\eta}^{\perp}\right) \quad\left(\boldsymbol{\xi}^{\perp}, \boldsymbol{\eta}^{\perp} \in H^{\perp}\right), \\
\omega\left(\gamma \boldsymbol{\xi}^{\perp}\right)=|\gamma| \omega\left(\boldsymbol{\xi}^{\perp}\right) \quad\left(\boldsymbol{\xi}^{\perp} \in H^{\perp}, \gamma \in \mathbb{R}\right) .
\end{gathered}
$$

Lemma 8.2. Suppose that $\omega: H^{\perp} \rightarrow \mathbb{R}$ satisfies (i)-(iii). Then there exists a basis $\boldsymbol{b}_{1}^{\perp}, \ldots, \boldsymbol{b}_{s}^{\perp}$ of $\Gamma$ such that for $\lambda_{1}, \ldots, \lambda_{s} \in \mathbb{R}$,

$$
\omega\left(\lambda_{1} \boldsymbol{b}_{1}^{\perp}+\ldots+\lambda_{s} \boldsymbol{b}_{s}^{\perp}\right) \geq 4^{-s} \max _{i}\left(\left|\lambda_{i}\right| \omega\left(\boldsymbol{b}_{i}^{\perp}\right)\right) .
$$

Pr o of. In the case when $\Gamma=\mathbb{Z}^{s}$, this is Proposition 4.2 of Schlickewei [5]. The general case follows easily. Let $\varphi: \Gamma \rightarrow \mathbb{Z}^{s}$ be an isomorphism of 
$\mathbb{Z}$-modules, and extend $\varphi$ to an isomorphism $H^{\perp} \rightarrow \mathbb{R}^{s}$. Define the function $\omega^{*}$ on $\mathbb{R}^{s}$ by

$$
\omega^{*}(\boldsymbol{x})=\omega\left(\varphi^{-1}(\boldsymbol{x})\right)
$$

Then $\omega^{*}$ satisfies (i)-(iii) with $\Gamma=\mathbb{Z}^{s}$. By Schlickewei's result, there exists a basis $\varepsilon_{1}, \ldots, \varepsilon_{s}$ of $\mathbb{Z}^{s}$ such that for any $\lambda_{1}, \ldots, \lambda_{s} \in \mathbb{R}$,

$$
\omega^{*}\left(\lambda_{1} \varepsilon_{1}+\ldots+\lambda_{s} \varepsilon_{s}\right) \geq 4^{-s} \max _{i}\left(\left|\lambda_{i}\right| \omega^{*}\left(\varepsilon_{i}\right)\right) .
$$

Then the basis $\boldsymbol{b}_{1}^{\perp}, \ldots, \boldsymbol{b}_{s}^{\perp}$ of $\Gamma$ given by $\boldsymbol{b}_{i}^{\perp}=\varphi^{-1}\left(\varepsilon_{i}\right)(i=1, \ldots, s)$ satisfies the conditions of the lemma.

Lemma 8.3. There exists a basis $\boldsymbol{a}_{1}^{\perp}, \ldots, \boldsymbol{a}_{s}^{\perp}$ of $\Gamma$ such that for $\lambda_{1}, \ldots, \lambda_{n}$ $\in \mathbb{R}$

$$
\left|\lambda_{1} \boldsymbol{a}_{1}^{\perp}+\ldots+\lambda_{s} \boldsymbol{a}_{s}^{\perp}\right| \leq s^{2} \max _{i}\left|\lambda_{i}\right| .
$$

Pr o o f. Let $\boldsymbol{e}_{1}, \ldots, \boldsymbol{e}_{n}$ be the standard basis for $\mathbb{R}^{n}$. Then the projections $\boldsymbol{e}_{1}^{\perp}, \ldots, \boldsymbol{e}_{n}^{\perp}$ span $\Gamma$ over $\mathbb{R}$. For each $i,\left|\boldsymbol{e}_{i}^{\perp}\right| \leq 1$. By a fact from the Geometry of Numbers (see, for example, Lemma 8 of $[1, \mathrm{Ch}$. V]), there exists a basis $\boldsymbol{a}_{1}^{\perp}, \ldots, \boldsymbol{a}_{s}^{\perp}$ of $\Gamma$ such that $\left|\boldsymbol{a}_{i}^{\perp}\right| \leq s \max \left|\boldsymbol{e}_{i}^{\perp}\right| \leq s(i=1, \ldots, s)$. Then

$$
\left|\lambda_{1} \boldsymbol{a}_{1}^{\perp}+\ldots+\lambda_{s} \boldsymbol{a}_{s}^{\perp}\right| \leq s \max \left|\lambda_{i} \boldsymbol{a}_{i}^{\perp}\right| \leq s^{2} \max \left|\lambda_{i}\right|,
$$

as required.

Let $\left\{\boldsymbol{b}_{i}^{\perp}\right\}_{i=1}^{s}$ and $\left\{\boldsymbol{a}_{i}^{\perp}\right\}_{i=1}^{s}$ be the bases of $\Gamma$ given by Lemmas 8.2 and 8.3. Suppose that $\boldsymbol{\xi}^{\perp}=\lambda_{1} \boldsymbol{a}_{1}^{\perp}+\ldots+\lambda_{s} \boldsymbol{a}_{s}^{\perp} \in \Gamma$. Then, using Lemmas 8.2 and 8.3 along with (i), we obtain

$$
\begin{aligned}
\omega\left(\lambda_{1} \boldsymbol{b}_{1}^{\perp}+\ldots+\lambda_{s} \boldsymbol{b}_{s}^{\perp}\right) & \geq 4^{-s} \max _{i}\left(\left|\lambda_{i}\right| \omega\left(\boldsymbol{b}_{i}^{\perp}\right)\right) \geq 4^{-s}\left(21 d^{3}\right)^{-1} \max _{i}\left|\lambda_{i}\right| \\
& \geq 4^{-s} s^{-2}\left(21 d^{3}\right)^{-1}\left|\boldsymbol{\xi}^{\perp}\right| .
\end{aligned}
$$

For $i=1, \ldots, s$ fix vectors $\boldsymbol{a}_{i}, \boldsymbol{b}_{i} \in \mathbb{Z}^{n}$ which project onto $\boldsymbol{a}_{i}^{\perp}, \boldsymbol{b}_{i}^{\perp}$, respectively. Let $\left\{\boldsymbol{c}_{s+1}, \ldots, \boldsymbol{c}_{n}\right\}$ be a basis for the lattice of integer points on $H$. We claim that $\boldsymbol{a}_{1}, \ldots, \boldsymbol{a}_{s}, \boldsymbol{c}_{s+1}, \ldots, \boldsymbol{c}_{n}$ form a basis of $\mathbb{Z}^{n}$. To see this, let $\boldsymbol{x}$ be a given element of $\mathbb{Z}^{n}$. If $\boldsymbol{x}^{\perp}=\sum_{i=1}^{s} \varrho_{i} \boldsymbol{a}_{i}^{\perp}$ with $\varrho_{i} \in \mathbb{Z}$, then set $\boldsymbol{y}=\boldsymbol{x}-\sum_{i=1}^{s} \varrho_{i} \boldsymbol{a}_{i}$. Since $\boldsymbol{y}^{\perp}=\mathbf{0}$, we conclude that $\boldsymbol{y}$ is an integer point on $H$, and our claim follows. Similarly, $\boldsymbol{b}_{1}, \ldots, \boldsymbol{b}_{s}, \boldsymbol{c}_{s+1}, \ldots, \boldsymbol{c}_{n}$ form a basis of $\mathbb{Z}^{n}$, and we may define a transformation $\Theta \in \mathrm{GL}(n, \mathbb{Z})$ by

$$
\begin{aligned}
& \Theta\left(\boldsymbol{a}_{i}\right)=\boldsymbol{b}_{i} \quad(i=1, \ldots, s), \\
& \Theta\left(\boldsymbol{c}_{i}\right)=\boldsymbol{c}_{i} \quad(i=s+1, \ldots, n) .
\end{aligned}
$$

It is clear that $\Theta$ fixes $H$ pointwise. Using (8.7), we see that for $\boldsymbol{\xi} \in \mathbb{R}^{n}$, we have

$$
\omega(\Theta(\boldsymbol{\xi}))=\omega\left(\Theta(\boldsymbol{\xi})^{\perp}\right) \geq c_{1}\left|\boldsymbol{\xi}^{\perp}\right|,
$$


where

$$
c_{1}=4^{-s} s^{-2}\left(21 d^{3}\right)^{-1} .
$$

By the discussion in Section 6, we may apply the transformation $\Theta$. Recall that this entails replacing the vectors $\boldsymbol{\alpha}_{l}$ with vectors $\boldsymbol{\beta}_{l}$ satisfying

$$
\boldsymbol{\beta}_{l}^{x}=\boldsymbol{\alpha}_{l}^{\Theta x} .
$$

The function $\omega=\omega_{\alpha}$ was defined in terms of the $\boldsymbol{\alpha}_{l}$. Similarly, define $\omega_{\beta}$ in terms of the $\boldsymbol{\beta}_{l}$. Then for $\boldsymbol{\xi} \in \mathbb{R}^{n}$, we have

$$
\omega_{\beta}(\boldsymbol{\xi})=\omega_{\alpha}(\Theta \boldsymbol{\xi}) \geq c_{1}\left|\boldsymbol{\xi}^{\perp}\right| .
$$

We may therefore assume from this point forward that

$$
\omega(\boldsymbol{\xi})=\omega^{\mathcal{P}}(\boldsymbol{\xi}) \geq c_{1}\left|\boldsymbol{\xi}^{\perp}\right| \quad\left(\boldsymbol{\xi} \in \mathbb{R}^{n}\right) .
$$

9. Construction of linear forms. Recall that $\mathcal{L}(T)$ denotes the space of linear forms vanishing on $T$. As mentioned in Section 5 , the plan is to apply Theorem 3.1 to equations of the form (3.2) defined by certain minimal forms $L \in \mathcal{L}(T)$. Since we must apply transformations to the vector $\boldsymbol{x}$, we require forms with certain special properties. Here we outline the construction of such forms developed in Section 16 of [7] and quote several results proved in that work.

Suppose that $\mathcal{P}$ is not the partition into singletons, and that

$$
\mathcal{P} \prec T \text {. }
$$

For $\lambda \in \mathcal{P}$ let $\mathcal{L}_{\lambda}(T)$ consist of forms $L \in \mathcal{L}(T)$ with $\mathcal{B}(L) \subseteq \lambda$.

Now let $\lambda \in \mathcal{P}$ with $|\lambda|>1$ be given. For simplicity, suppose that $\lambda=\{1, \ldots, r\}$. We will construct a partition of $\lambda, \lambda=\bigcup_{j=1}^{t} \mu_{j}$, into nonempty subsets $\mu_{j}$, as well as forms $L_{1}, \ldots, L_{t}$ in $\mathcal{L}_{\lambda}(T)$.

We call a form $L \in \mathcal{L}_{\lambda}(T)$ 1-stable if $|\mathcal{B}(L)|>1$ and $L$ cannot be written as a sum $L=L^{\prime}+L^{\prime \prime}$ where $L^{\prime}$ and $L^{\prime \prime}$ are non-zero, lie in $\mathcal{L}_{\lambda}(T)$, and have $\mathcal{B}\left(L^{\prime}\right) \cap \mathcal{B}\left(L^{\prime \prime}\right)=\emptyset$. There are 1-stable forms; if there were not then every form in $\mathcal{L}_{\lambda}(T)$ would be a sum of forms whose sets $\mathcal{B}$ have cardinality one, so that if $\mathcal{Q}$ were obtained from $\mathcal{P}$ by breaking $\lambda$ into the singletons $\{1\}, \ldots,\{r\}$ we would have $T(\mathcal{Q})=T$, against (9.1). We may therefore choose a set $\mu_{1} \subseteq \lambda$ with minimal cardinality such that there is a 1-stable form $L$ with $\mathcal{B}(L)=\mu_{1}$. Notice that $\left|\mu_{1}\right|>1$.

Suppose that $j>1$ and that subsets $\mu_{1}, \ldots, \mu_{j-1}$ of $\lambda$ have been constructed. Set

$$
\nu_{j-1}=\bigcup_{i=1}^{j-1} \mu_{i} .
$$

If $\nu_{j-1}=\lambda$ we set $t=j-1$, and our construction is finished. Otherwise let $\bar{\nu}_{j-1}$ be the complement of $\nu_{j-1}$ in $\lambda$. We call a form $L \in \mathcal{L}_{\lambda}(T) j$-stable if 
$L$ cannot be written as $L=L^{\prime}+L^{\prime \prime}$ where $\mathcal{B}\left(L^{\prime}\right) \subseteq \nu_{j-1}$ and $\mathcal{B}\left(L^{\prime \prime}\right) \subseteq \bar{\nu}_{j-1}$. $j$-stable forms do exist, for otherwise every form $L \in \mathcal{L}_{\lambda}(T)$ could be written as a sum $L=L^{\prime}+L^{\prime \prime}$ with $L^{\prime}, L^{\prime \prime}$ as above. Then if $\mathcal{Q}$ is obtained from $\mathcal{P}$ by dividing $\lambda$ into $\nu_{j-1}$ and $\bar{\nu}_{j-1}$, we would have $T(\mathcal{Q})=T$, contradicting (9.1).

We may therefore choose $\mu_{j} \subseteq \bar{\nu}_{j-1}$ of minimal cardinality such that there is a $j$-stable form $L$ with

$$
\mathcal{B}(L) \cap \bar{\nu}_{j-1}=\mu_{j} .
$$

It is clear that $\mu_{j} \neq \emptyset$. Continuing in this manner, we obtain sets $\mu_{1}, \ldots, \mu_{t}$ which partition $\lambda$.

Now renumber the elements of $\lambda$ so that

$$
\mu_{j}=\left\{r_{j-1}+1, \ldots, r_{j}\right\} \quad(1 \leq j \leq t)
$$

with $0=r_{0}<r_{1}<\ldots<r_{t}=r$. Recall the definition (given in Section 7) of the leading monomials $M_{1}(L), \ldots, M_{k}(L)$. Given forms $L, L^{\prime} \in \mathcal{L}_{\lambda}(T)$, write $L^{\prime}<L$ if

$$
M_{s}\left(L^{\prime}\right)<M_{s}(L), \quad M_{s+1}\left(L^{\prime}\right)=M_{s+1}(L), \quad \ldots, \quad M_{r}\left(L^{\prime}\right)=M_{r}(L)
$$

for some $s$ (this should not be confused with our earlier ordering of monomials).

By the construction, for every $j, 1 \leq j \leq t$, there exist $j$-stable forms $L_{j}$ satisfying (9.2), where we define $\nu_{0}=\emptyset, \bar{\nu}_{0}=\lambda$. A form $L_{j}$ is $j$-proper if it is minimal with respect to $<$ among all $j$-stable forms with (9.2). Since the ordering $<$ depends only on the leading monomials, $j$-proper forms may not be uniquely determined. If, however, both $L_{j}$ and $L_{j}^{\prime}$ are $j$-proper, then

$$
\left(M_{1}\left(L_{j}\right), \ldots, M_{r_{j}}\left(L_{j}\right)\right)=\left(M_{1}\left(L_{j}^{\prime}\right), \ldots, M_{r_{j}}\left(L_{j}^{\prime}\right)\right) .
$$

We shall require several results from [7].

Lemma 9.1 ([7, Lemmas 16.1, 16.2]). (1) Suppose that $L_{j}$ and $L_{j}^{\prime}$ are $j$-proper. Then their tuples of leading coefficients are proportional.

(2) Let $L_{j}$ be a j-proper form with $\left|\mathcal{A}\left(L_{j}\right)\right|$ as small as possible. Then $L_{j}$ is a minimal form.

Now let $\mathcal{P}$ be a partition as in Theorem 4.1, and for every $\lambda \in \mathcal{P}$ with $|\lambda|>1$ construct $j$-proper forms $L_{\lambda j}, j=1, \ldots, t$, with $t=t(\lambda) \leq|\lambda|$, as above. This set of forms will be fixed for the remainder.

Lemma 9.2 ([7, Lemma 17.1]). The forms $L_{\lambda j}$ satisfy the following property: For every $\lambda \in \mathcal{P}$ and every $l, m \in \lambda$ there is a chain of forms $L_{\lambda j(1)}, \ldots$, $L_{\lambda j(q)}$ with $q \leq t(\lambda), l \in \mathcal{B}\left(L_{\lambda j(1)}\right)$, and $m \in \mathcal{B}\left(L_{\lambda j(q)}\right)$, and such that

$$
\mathcal{B}\left(L_{\lambda j(i)}\right) \cap \mathcal{B}\left(L_{\lambda j(i+1)}\right) \neq \emptyset \quad(1 \leq i<q) .
$$


To ease notation, we write Max to denote the maximum over all pairs $l, m$ with $l, m \in \mathcal{B}\left(L_{\lambda j}\right)$ for some $\lambda, j$. Recall the definition (8.3) of the functions $\psi_{l m}$, and for $\boldsymbol{\xi} \in \mathbb{R}^{n}$ define

$$
\mathcal{X}^{\mathcal{P}}(\boldsymbol{\xi})=\operatorname{Max} \psi_{l m}(\boldsymbol{\xi}) .
$$

We shall require the next lemma in Section 10.

LEMma 9.3. For $\boldsymbol{\xi} \in \mathbb{R}^{n}$, we have

$$
\mathcal{X}^{\mathcal{P}}(\boldsymbol{\xi}) \geq c_{2}\left|\boldsymbol{\xi}^{\perp}\right|
$$

where

$$
c_{2}=\left(21 k d^{3} s^{2} 4^{s}\right)^{-1} .
$$

Proof. Suppose that $\boldsymbol{\xi} \in \mathbb{R}^{n}$. By (8.6) and (8.9), $\psi_{l m}(\boldsymbol{\xi}) \geq c_{1}\left|\boldsymbol{\xi}^{\perp}\right|$ for some $l, m$ with $l \stackrel{\mathcal{P}}{\sim} m$; say $l, m \in \lambda$ where $\lambda \in \mathcal{P}$. Let $L_{\lambda j(1)}, \ldots, L_{\lambda j(q)}$ be the chain of forms given by Lemma 9.2, and let $l(i)$ lie in the set (9.3). Then, using (8.5), we have

$$
c_{1}\left|\boldsymbol{\xi}^{\perp}\right| \leq \psi_{l m}(\boldsymbol{\xi}) \leq \psi_{l, l(1)}(\boldsymbol{\xi})+\ldots+\psi_{l(q-1), m}(\boldsymbol{\xi}) .
$$

The lemma follows since $c_{2}=c_{1} / k$ and $q \leq k$.

10. Preparing for the second transformation. Recall that we have fixed a set of forms $L_{\lambda j}$. In this section we use a translation $\boldsymbol{x} \mapsto \boldsymbol{x}-\boldsymbol{u}$ in order to control the height of the coefficients of these forms. Since $\mathcal{P}$ is fixed, we will simplify our notation by writing $\mathcal{X}=\mathcal{X}^{\mathcal{P}}$. When $l \stackrel{\mathcal{P}}{\sim} m$ we have $\psi_{l m}\left(\boldsymbol{\xi}^{\perp}\right)=\psi_{l m}(\boldsymbol{\xi})$ for any $\boldsymbol{\xi} \in \mathbb{R}^{n}$, therefore also $\mathcal{X}\left(\boldsymbol{\xi}^{\perp}\right)=\mathcal{X}(\boldsymbol{\xi})$. Now $\mathcal{X}$ is a non-negative function which satisfies

$$
\begin{aligned}
\mathcal{X}\left(\boldsymbol{\xi}^{\perp}+\boldsymbol{\eta}^{\perp}\right) \leq \mathcal{X}\left(\boldsymbol{\xi}^{\perp}\right)+\mathcal{X}\left(\boldsymbol{\eta}^{\perp}\right), \quad \mathcal{X}\left(\gamma \boldsymbol{\xi}^{\perp}\right)=|\gamma| \mathcal{X}\left(\boldsymbol{\xi}^{\perp}\right) \\
\quad\left(\gamma \in \mathbb{R}, \boldsymbol{\xi}^{\perp}, \boldsymbol{\eta}^{\perp} \in H^{\perp}\right) .
\end{aligned}
$$

In view of Lemma 9.3, we may conclude that the set $\mathbb{X} \subset H^{\perp}$ defined by

$$
\mathbb{X}=\left\{\boldsymbol{\xi}^{\perp} \in H^{\perp}: \mathcal{X}\left(\boldsymbol{\xi}^{\perp}\right) \leq 1\right\}
$$

is a convex, symmetric, compact set which contains $\mathbf{0}$ in its interior (see, for example, Chapter 4 of [1]).

We will now consider points $(\boldsymbol{\xi}, \zeta) \in \mathbb{R}^{n+1}=\mathbb{R}^{n} \times \mathbb{R}$. Recall the definitions of $g_{v}^{l m}$ in (8.2) and of the leading monomials $b_{l}$ at the end of Section 7. Then, for $l, m \in \mathcal{B}\left(L_{\lambda j}\right)$, define

$$
\begin{aligned}
c_{\lambda j v}^{l m} & =\log \left\|b_{l}\left(L_{\lambda j}\right) / b_{m}\left(L_{\lambda j}\right)\right\|_{v} \\
\widetilde{g}_{\lambda j v}^{l m}(\boldsymbol{\xi}, \zeta) & =g_{v}^{l m}(\boldsymbol{\xi})+c_{\lambda j v}^{l m} \zeta \\
\widetilde{\psi}_{\lambda j}^{l m}(\boldsymbol{\xi}, \zeta) & =\sum_{v} \max \left(0, \widetilde{g}_{\lambda j v}^{l m}(\boldsymbol{\xi}, \zeta)\right)=\frac{1}{2} \sum_{v}\left|\widetilde{g}_{\lambda j v}^{l m}(\boldsymbol{\xi}, \zeta)\right|,
\end{aligned}
$$




$$
\widetilde{\mathcal{X}}(\boldsymbol{\xi}, \zeta)=\operatorname{Max} \widetilde{\psi}_{\lambda j}^{l m}(\boldsymbol{\xi}, \zeta)
$$

Notice that for $\boldsymbol{x} \in \mathbb{Z}^{n}$ we have

$$
\begin{aligned}
\widetilde{\mathcal{X}}(\boldsymbol{x}, 1) & =\operatorname{Max} h\left(b_{l}\left(L_{\lambda j}\right) \boldsymbol{\alpha}_{l}^{x}, b_{m}\left(L_{\lambda j}\right) \boldsymbol{\alpha}_{m}^{x}\right) \\
& =\operatorname{Max} h\left(b_{l}\left(L_{\lambda j}\right) \boldsymbol{\alpha}_{l}^{x} / b_{m}\left(L_{\lambda j}\right) \boldsymbol{\alpha}_{m}^{x}\right) .
\end{aligned}
$$

Also notice that for any $\boldsymbol{\xi} \in \mathbb{R}^{n}$ we have $\tilde{\mathcal{X}}(\boldsymbol{\xi}, 0)=\mathcal{X}(\boldsymbol{\xi})$ and $\tilde{\mathcal{X}}\left(\boldsymbol{\xi}^{\perp}, \zeta\right)=$ $\widetilde{\mathcal{X}}(\boldsymbol{\xi}, \zeta)$. Let $\widetilde{\mathbb{X}} \subset H^{\perp} \times \mathbb{R}$ be the set of points $\left(\boldsymbol{\xi}^{\perp}, \zeta\right)$ such that $\widetilde{\mathcal{X}}\left(\boldsymbol{\xi}^{\perp}, \zeta\right) \leq 1$. $\widetilde{\mathcal{X}}$ enjoys properties analogous to those in $(10.1)$; therefore $\widetilde{\mathbb{X}}$ is convex, symmetric, closed, and has $(\mathbf{0}, 0)$ in its interior. However, $\widetilde{\mathbb{X}}$ may be unbounded.

We extract the following from the proof of Lemma 15.1 of [7].

Lemma 10.1. There exists a fixed $\boldsymbol{\xi}_{1} \in \mathbb{R}^{n}$ such that for any $\boldsymbol{\xi} \in \mathbb{R}^{n}$,

$$
\mathcal{X}(\boldsymbol{\xi}) \leq 2 \tilde{\mathcal{X}}\left(\boldsymbol{\xi}+\boldsymbol{\xi}_{1}, 1\right) .
$$

Proof. If $\widetilde{\mathbb{X}}$ is unbounded, then there exists $\left(\boldsymbol{\xi}_{0}^{\perp}, \zeta_{0}\right) \neq(\mathbf{0}, 0)$ with $\widetilde{\mathcal{X}}\left(\boldsymbol{\xi}_{0}^{\perp}, \zeta_{0}\right)=0$. Since $\mathbb{X}$ is bounded, we must have $\zeta_{0} \neq 0$; by homogeneity we may choose $\left(\boldsymbol{\xi}_{1}^{\perp}, 1\right)$ with $\widetilde{\mathcal{X}}\left(\boldsymbol{\xi}_{1}^{\perp}, 1\right)=0$. On the other hand, when $\widetilde{\mathbb{X}}$ is bounded, hence compact, choose $\left(\boldsymbol{\xi}_{0}^{\perp}, \zeta_{0}\right) \in \widetilde{\mathbb{X}}$ with $\zeta_{0}$ maximal. Rewrite $\boldsymbol{\xi}_{0}^{\perp}=\zeta_{0} \boldsymbol{\xi}_{1}^{\perp}$, so that $\zeta_{0}\left(\boldsymbol{\xi}_{1}^{\perp}, 1\right) \in \widetilde{\mathbb{X}}$.

Now suppose that $\left(\boldsymbol{\xi}^{\perp}, \zeta\right) \in \widetilde{\mathbb{X}}$. When $\widetilde{\mathbb{X}}$ is unbounded, $\zeta\left(\boldsymbol{\xi}_{1}^{\perp}, 1\right) \in \widetilde{\mathbb{X}}$, but this holds also when $\widetilde{\mathbb{X}}$ is bounded, since $|\zeta| \leq \zeta_{0}$ in that case. Taking the difference, we see that $\left(\boldsymbol{\xi}^{\perp}-\zeta \boldsymbol{\xi}_{1}^{\perp}, 0\right) \in 2 \widetilde{\mathbb{X}}$, from which $\boldsymbol{\xi}^{\perp}-\zeta \boldsymbol{\xi}_{1}^{\perp} \in 2 \mathbb{X}$. In other words, $\left(\boldsymbol{\xi}^{\perp}, \zeta\right) \in \widetilde{\mathbb{X}}$ implies that $\boldsymbol{\xi}^{\perp}-\zeta \boldsymbol{\xi}_{1}^{\perp} \in 2 \mathbb{X}$. By reason of homogeneity, $\mathcal{X}\left(\boldsymbol{\xi}^{\perp}-\zeta \boldsymbol{\xi}_{1}^{\perp}\right) \leq 2 \widetilde{\mathcal{X}}\left(\boldsymbol{\xi}^{\perp}, \zeta\right)$. We may conclude that $\mathcal{X}\left(\boldsymbol{\xi}^{\perp}\right) \leq$ $2 \widetilde{\mathcal{X}}\left(\boldsymbol{\xi}^{\perp}+\zeta \boldsymbol{\xi}_{1}^{\perp}, \zeta\right)$ for any $\left(\boldsymbol{\xi}^{\perp}, \zeta\right) \in H^{\perp} \times \mathbb{R}$. The lemma follows upon setting $\zeta=1$.

Now let $\boldsymbol{\xi}_{1}$ be as in Lemma 10.1 , choose $\boldsymbol{u} \in \mathbb{Z}^{n}$ such that $\left|\boldsymbol{u}+\boldsymbol{\xi}_{1}\right| \leq$ $\sqrt{n} / 2$, and set $\boldsymbol{\mu}=\boldsymbol{\xi}_{1}+\boldsymbol{u}$. Then Lemmas 9.3 and 10.1 show that for $\boldsymbol{x} \in \mathbb{Z}^{n}$ we have

$$
\begin{aligned}
\widetilde{\mathcal{X}}(\boldsymbol{x}-\boldsymbol{u}, 1) & =\widetilde{\mathcal{X}}\left(\boldsymbol{x}-\boldsymbol{\mu}+\boldsymbol{\xi}_{1}, 1\right) \\
& \geq \frac{1}{2} \mathcal{X}(\boldsymbol{x}-\boldsymbol{\mu}) \geq \frac{1}{2} c_{2}\left|\boldsymbol{x}^{\perp}-\boldsymbol{\mu}^{\perp}\right| \geq \frac{1}{2} c_{2}\left(\left|\boldsymbol{x}^{\perp}\right|-\sqrt{n} / 2\right) .
\end{aligned}
$$

In view of (10.2) we have proved the following

Lemma 10.2. There exists $\boldsymbol{u} \in \mathbb{Z}^{n}$ such that for any $\boldsymbol{x} \in \mathbb{Z}^{n}$,

$$
\operatorname{Max} h\left(b_{l}\left(L_{\lambda j}\right) \boldsymbol{\alpha}_{l}^{-u} \boldsymbol{\alpha}_{l}^{x}, b_{m}\left(L_{\lambda j}\right) \boldsymbol{\alpha}_{m}^{-u} \boldsymbol{\alpha}_{m}^{x}\right) \geq \frac{1}{2} c_{2}\left(\left|\boldsymbol{x}^{\perp}\right|-\sqrt{n} / 2\right) .
$$

11. Applying the second transformation. Let $u \in \mathbb{Z}^{n}$ be the point given in Lemma 10.2, and apply the transformation $\phi \boldsymbol{x}=\boldsymbol{x}-\boldsymbol{u}$ to the 
variable vector $\boldsymbol{x}$. As in Section 6 , this induces an invertible linear transformation $\widehat{\phi}$ on $K^{A}$, which, by (6.1), satisfies

$$
\boldsymbol{\xi}(\phi \boldsymbol{x})=\widehat{\phi}(\boldsymbol{\xi}(\boldsymbol{x})) \text {. }
$$

(Notice that such a transformation does not involve replacing the exponential bases $\boldsymbol{\alpha}_{l}$.)

Now define forms $\widehat{L}_{\lambda j}$ on $K^{A}$ by

$$
\widehat{L}_{\lambda j}(\boldsymbol{\xi}(\boldsymbol{x}))=L_{\lambda j}(\boldsymbol{\xi}(\boldsymbol{x}-\boldsymbol{u})) .
$$

By (11.1) we see that $\widehat{L}_{\lambda j} \in \mathcal{L}\left(\widehat{\phi}^{-1} T\right)$. Moreover, since $\mathcal{B}\left(\widehat{L}_{\lambda j}\right)=\mathcal{B}\left(L_{\lambda j}\right)$, these new forms are again $j$-stable, now with respect to the subspace $\widehat{\phi}^{-1} T$. Finally, since the leading monomials of $\widehat{L}_{\lambda j}$ are the same as those of $L_{\lambda j}$, we see that the new forms are in fact $j$-proper.

If $b_{l}\left(L_{\lambda j}\right)$ was a leading coefficient of $L_{\lambda j}$, then $b_{l}\left(L_{\lambda j}\right) \boldsymbol{\alpha}_{l}^{-u}$ is a leading coefficient of $\widehat{L}_{\lambda j}$. By Lemma 10.2 we obtain

$$
\operatorname{Max} h\left(b_{l}\left(\widehat{L}_{\lambda j}\right) \boldsymbol{\alpha}_{l}^{x}, b_{m}\left(\widehat{L}_{\lambda j}\right) \boldsymbol{\alpha}_{m}^{x}\right) \geq \frac{1}{2} c_{2}\left(\left|\boldsymbol{x}^{\perp}\right|-\sqrt{n} / 2\right) \quad\left(\boldsymbol{x} \in \mathbb{Z}^{n}\right) .
$$

We now replace the forms $\widehat{L}_{\lambda j}$ with $j$-proper forms $\widetilde{L}_{\lambda j} \in \mathcal{L}\left(\widehat{\phi}^{-1} T\right)$ whose sets $\mathcal{A}\left(\widetilde{L}_{\lambda j}\right)$ are as small as possible. By Lemma 9.1, each form $\widetilde{L}_{\lambda j}$ is minimal, and has leading coefficients which are proportional to those of $\widehat{L}_{\lambda j}$. Therefore (11.2) still holds when we replace $\widehat{L}_{\lambda j}$ with $\widetilde{L}_{\lambda j}$.

For any form $L$ with coefficients $b_{l M}$ as in (7.1), let $h_{L E}(\boldsymbol{x})$ denote the height of the vector with components

$$
b_{l M} \boldsymbol{\alpha}_{l}^{x} \quad((l, M) \in \mathcal{A}(L)) .
$$

It is clear that we have

$$
\max _{\lambda, j} h_{\widetilde{L}_{\lambda j} E}(\boldsymbol{x}) \geq \operatorname{Max} h\left(b_{l}\left(\widetilde{L}_{\lambda j}\right) \boldsymbol{\alpha}_{l}^{x}, b_{m}\left(\widetilde{L}_{\lambda j}\right) \boldsymbol{\alpha}_{m}^{x}\right) .
$$

To ease notation we shall write $L_{\lambda j}$ for $\widetilde{L}_{\lambda j}$. In view of (11.2) and (11.3) we have proved the following

Lemma 11.1. We may assume that the forms $L_{\lambda j}$ are minimal. Further, we may suppose that for $\boldsymbol{x} \in \mathbb{Z}^{n}$,

$$
\max _{\lambda, j} h_{L_{\lambda j} E}(\boldsymbol{x}) \geq \frac{1}{2} c_{2}\left(\left|\boldsymbol{x}^{\perp}\right|-\sqrt{n} / 2\right) .
$$

12. Proof of Theorem 4.1. Recall the definition of $\mathfrak{X}(T, \mathcal{P})$ given in Section 4. Using Lemma 11.1, we may divide solutions $\boldsymbol{x} \in \mathfrak{X}(T, \mathcal{P})$ into classes $\mathcal{C}_{\lambda j}$ with $\boldsymbol{x} \in \mathcal{C}_{\lambda j}$ if

$$
h_{L_{\lambda j} E}(\boldsymbol{x}) \geq \frac{1}{2} c_{2}\left(\left|\boldsymbol{x}^{\perp}\right|-\sqrt{n} / 2\right) .
$$

There are at most $A$ classes. To ease notation, we fix a form $L=L_{\lambda j}$ and the corresponding class $\mathcal{C}=\mathcal{C}_{\lambda j}$. Recall the definitions (1.3), (1.4) of $c_{0}$ and $\mathcal{S}$. 
Vectors $\boldsymbol{x} \in \mathcal{C}$ satisfying

$$
\boldsymbol{x} \notin \mathcal{S}
$$

have $\left|\boldsymbol{x}^{\perp}\right| \geq c_{0}>\sqrt{n}$, so that by (12.1), such $\boldsymbol{x}$ have

$$
h_{L E}(\boldsymbol{x}) \geq c_{3}\left|\boldsymbol{x}^{\perp}\right|
$$

where

$$
c_{3}=\frac{1}{4} c_{2}=\left(84 k d^{3} s^{2} 4^{s}\right)^{-1} .
$$

We shall assume initially that (12.2), whence (12.3) holds.

For $\boldsymbol{x} \in \mathcal{C}$ we have $L(\boldsymbol{\xi}(\boldsymbol{x}))=0$. We restrict our attention to the vector $\boldsymbol{\xi}_{L}(\boldsymbol{x})$ with components $\xi_{l M}(\boldsymbol{x})$ where $(l, M) \in \mathcal{A}(L)$. Then $\boldsymbol{\xi}_{L}(\boldsymbol{x}) \in K^{a}$ with $a=|\mathcal{A}(L)| \geq 2$. With a slight abuse of notation, we will write

$$
L\left(\boldsymbol{\xi}_{L}(\boldsymbol{x})\right)=0 .
$$

This says that $\boldsymbol{\xi}_{L}(\boldsymbol{x})$ lies in a certain subspace $U$ of $K^{a}$ of codimension 1 .

Let $h_{L M}(\boldsymbol{x})$ be the height of the vector with components $M(\boldsymbol{x})(M \in$ $\left.\mathcal{M}_{l}, l \in \mathcal{B}(L)\right)$. Notice that for any $L$ the monomials which occur have total degree $\leq \max \left(\delta_{1}, \ldots, \delta_{k}\right) \leq A$. Therefore we always have

$$
h_{L M}(\boldsymbol{x}) \leq A \log ^{+}|\boldsymbol{x}| .
$$

Suppose first that $a=2$. Then the equation (12.5) takes the form

$$
b M(\boldsymbol{x}) \boldsymbol{\alpha}_{l}^{x}=b^{\prime} M^{\prime}(\boldsymbol{x}) \boldsymbol{\alpha}_{m}^{x},
$$

so that

$$
h_{L E}(\boldsymbol{x})=h_{L M}(\boldsymbol{x}) \leq A \log ^{+}|\boldsymbol{x}| .
$$

Together with (12.3) and (12.4) this gives

$$
\left|\boldsymbol{x}^{\perp}\right| \leq A \cdot 84 k d^{3} s^{2} 4^{s} \log ^{+}|\boldsymbol{x}| \leq B^{4} 2^{2 B+7} d^{3} \log ^{+}|\boldsymbol{x}|
$$

(here, and below, we use the fact that $\max (A, k, s) \leq B$, and that $84<2^{7}$ ). At this point it will be convenient to record a lemma.

Lemma 12.1. Suppose that $c \leq B^{6} 2^{2 B+9} d^{3}$ and that $\left|\boldsymbol{x}^{\perp}\right| \leq c \log ^{+}|\boldsymbol{x}|$. Then $\boldsymbol{x} \in \mathcal{S}$.

Proof. We operate under the hypothesis of the lemma. Suppose first that $\left|\boldsymbol{x}^{\perp}\right| \leq\left|\boldsymbol{x}^{H}\right|$. Then $|\boldsymbol{x}| \leq 2\left|\boldsymbol{x}^{H}\right|$, so that $\left|\boldsymbol{x}^{\perp}\right| \leq 2 c \log ^{+}\left|\boldsymbol{x}^{H}\right| \leq$ $c_{0} \log ^{+}\left|\boldsymbol{x}^{H}\right|$, and $\boldsymbol{x} \in \mathcal{S}$.

If, on the other hand, $\left|\boldsymbol{x}^{H}\right| \leq\left|\boldsymbol{x}^{\perp}\right|$, then $|\boldsymbol{x}| \leq 2\left|\boldsymbol{x}^{\perp}\right|$, whence $\left|\boldsymbol{x}^{\perp}\right| \leq$ $2 c \log ^{+}\left|\boldsymbol{x}^{\perp}\right|$. Then (since generally $\exp (t) \geq t^{2} / 2$ ), we obtain $\left|\boldsymbol{x}^{\perp}\right| \leq 8 c^{2} \leq$ $c_{0} \leq c_{0} \log ^{+}\left|\boldsymbol{x}^{H}\right|$, and again $\boldsymbol{x} \in \mathcal{S}$.

Applying this lemma, we see that $\boldsymbol{x}$ satisfying (12.7) lie in $\mathcal{S}$. Along with (12.2), this shows that $\mathcal{C} \subset \mathcal{S}$ when $a=2$. 
We will now assume that $a \geq 3$ (recall that we always have $a \leq A$ ). When $\boldsymbol{x} \in \mathcal{C}$, we have (12.5), which is of the form (3.2) studied in Theorem 3.1. To use this theorem, we need (3.3). In other words we need

$$
h_{L M}(\boldsymbol{x}) \leq \frac{1}{4 a^{2}} h_{L E}(\boldsymbol{x})
$$

which, using (12.3) and (12.6), will hold if

$$
A \log ^{+}|\boldsymbol{x}| \leq \frac{1}{4 A^{2}} c_{3}\left|\boldsymbol{x}^{\perp}\right| \text {. }
$$

After applying Theorem 3.1, we conclude that vectors $\boldsymbol{\xi}_{L}(\boldsymbol{x})$ with $\boldsymbol{x} \in \mathcal{C}$ and $\boldsymbol{x}$ satisfying (12.2), (12.8), lie in at most

$$
2^{30 a^{2}}\left(32 a^{2}\right)^{n} d^{3(n+a)} \leq 2^{34 B^{2}} d^{6 B}
$$

proper subspaces of $U$ (recall that $U$ is defined by (12.5)). Let $U^{\prime}$ be one of these subspaces. Then $U^{\prime}$ is defined by $L^{\prime}\left(\boldsymbol{\xi}_{L}\right)=0$ for a linear form $L^{\prime}$ which is not proportional to $L$. Since $\mathcal{A}\left(L^{\prime}\right) \subseteq \mathcal{A}(L)$, we may replace $L^{\prime}$ by $L^{\prime \prime}=L^{\prime}-\beta L$ with suitable $\beta \in K$ to ensure that $\mathcal{A}\left(L^{\prime \prime}\right) \varsubsetneqq \mathcal{A}(L)$. In other words, we may suppose that $\mathcal{A}\left(L^{\prime}\right) \varsubsetneqq \mathcal{A}(L)$. Now, since $L$ is minimal, we must have $L^{\prime} \notin \mathcal{L}(T)$. Therefore, whenever $\boldsymbol{\xi}_{L}(\boldsymbol{x}) \in U^{\prime}$, we may conclude that $\boldsymbol{\xi}(\boldsymbol{x})$ lies in a certain proper subspace $T^{\prime}$ of $T$. Since $\mathcal{B}\left(L^{\prime}\right) \subseteq \mathcal{B}(L) \subseteq \lambda$ for some $\lambda \in \mathcal{P}$, we have $T^{\prime}(\mathcal{P})=T^{\prime}$. Therefore, using the estimate (12.9), we see that points $\boldsymbol{x} \in \mathcal{C}$ satisfying (12.2) and (12.8) lie in a union $\bigcup_{i=1}^{C^{\prime}} \mathfrak{X}\left(T_{i}\right)$, where $C^{\prime}=2^{34 B^{2}} d^{6 B}$, and each $T_{i}$ is a proper subspace of $T$ with $T_{i}(\mathcal{P})=T_{i}$.

If, however, (12.8) is violated, then

$$
\left|\boldsymbol{x}^{\perp}\right| \leq 4 A^{3}\left(84 k d^{3} s^{2} 4^{s}\right) \log ^{+}|\boldsymbol{x}| \leq B^{6} 2^{2 B+9} d^{3} \log ^{+}|\boldsymbol{x}|,
$$

so that $\boldsymbol{x} \in \mathcal{S}$ by Lemma 12.1 .

When $a=2$, we have shown that our chosen class $\mathcal{C}$ is contained in $\mathcal{S}$. When $a \geq 3$, we have shown that $\mathcal{C}$ is contained in a set of the form $\mathcal{S} \cup \bigcup_{i=1}^{C^{\prime}} \mathfrak{X}\left(T_{i}\right)$. Theorem 4.1 follows easily, since there are at most $B$ possible classes.

Acknowledgments. I am indebted to Wolfgang Schmidt for his helpful comments on my work.

\section{References}

[1] J. W. S. Cassels, An Introduction to the Geometry of Numbers, Grundlehren Math. Wiss. 99, Springer, 1959.

[2] E. Dobrowolski, On a question of Lehmer and the number of irreducible factors of a polynomial, Acta Arith. 34 (1979), 391-401.

[3] M. Laurent, Équations diophantiennes exponentielles, Invent. Math. 78 (1984), 299327. 
[4] M. Laurent, Équations exponentielles-polynômes et suites récurrentes linéaires, II, J. Number Theory 31 (1989), 24-53.

[5] H. P. Schlickewei, Lower bounds for heights on finitely generated groups, Monatsh. Math. 123 (1997), 171-178.

[6] H. P. Schlickewei and W. M. Schmidt, On polynomial-exponential equations, Math. Ann. 296 (1993), 339-361.

[7] - - - The number of solutions of polynomial-exponential equations, Compositio Math., to appear.

Department of Mathematics

The Pennsylvania State University

University Park, Pennsylvania 16802-6401

U.S.A

E-mail: ahlgren@math.psu.edu

Received on 7.12.1995

and in revised form on 28.7.1998 